\title{
ST-segment elevation myocardial infarction: Evaluating the time interval from diagnosis to fibrinolysis at centres in the drainage area of Tygerberg Hospital, Cape Town, South Africa
}

\author{
B D Beyers, ${ }^{1} \mathrm{MB}$ ChB; A F Doubell, ${ }^{2} \mathrm{MB}$ ChB, FCP (SA), MMed (Internal Medicine), BSc Hons, PhD (Medical Biochemistry); \\ B Griffiths, ${ }^{2} \mathrm{MB}$ ChB, DA (SA), FCP (SA), MMed (Internal Medicine), Cert Cardiology (SA); T Jalavu, ${ }^{3}$ MB ChB \\ ${ }^{1}$ Robert Mangaliso Sobukwe Hospital, Kimberley, South Africa \\ ${ }^{2}$ Division of Cardiology, Department of Medicine, Faculty of Medicine and Health Sciences, Stellenbosch University and Tygerberg Hospital, \\ Cape Town, South Africa \\ ${ }^{3}$ National Health Laboratory Service, Stellenbosch University and Tygerberg Hospital, Cape Town, South Africa
}

Corresponding author: B D Beyers (briandeonbeyers@icloud.com)

Background. ST-segment elevation myocardial infarction (STEMI) is one of the main contributors to morbidity and mortality in South Africa (SA). Timeous intervention by means of percutaneous coronary intervention (PCI) or fibrinolysis can significantly improve the outcome of STEMI.

Objectives. To determine the median time interval between diagnosis and fibrinolysis in patients presenting to centres within the drainage area of Tygerberg Hospital, Cape Town, SA, and compare it with the European Society of Cardiology (ESC) recommendation of 10 minutes. Methods. A retrospective medical record review of patients presenting to the abovementioned centres between 1 March 2017 and 28 February 2018 was performed. The primary presenting centre, time between diagnosis and fibrinolysis and discharge medication were recorded, in addition to other relevant demographic information.

Results. A total of 492 patients were identified, of whom 447 were included in the study. Three hundred and eighteen patients received fibrinolysis, of whom 18 (5.7\%) were treated within 10 minutes of diagnosis. The median time interval between diagnosis and fibrinolysis was 67 (interquartile range (IQR) 32.5 - 122.5) minutes.

Conclusions. Most patients received fibrinolysis $>10$ minutes after diagnosis, which indicates suboptimal therapy when compared with the ESC guidelines. Future studies should investigate the factors prolonging this therapeutic delay.

S Afr Med J 2020;110(4):327-331. https://doi.org/10.7196/SAMJ.2020.v110i4.14330

Cardiovascular disease, specifically ischaemic heart disease (IHD), is the main cause of death in developed countries, as cited by a 2018 World Health Organization (WHO) report. ${ }^{[1]}$ According to Statistics South Africa (Stats SA), ${ }^{[2]}$ the main contributors to mortality in SA in 2016 were disorders of the circulatory system $(18.5 \%)$, followed by communicable diseases (18.2\%). When evaluating the trend in mortality rates since 2013 , it was found that deaths due to communicable diseases saw a $3 \%$ decline (from $22.5 \%$ ), while disorders of the circulatory system saw a $1.1 \%$ increase in deaths in 2015 . These figures confirm the findings of the report $^{[2]}$ that there is an epidemiological shift away from communicable diseases towards non-communicable diseases.

In the report by Stats SA, ${ }^{[2]} \mathrm{IHD}$ was found to be the third most common natural cause of death (6.0\%) in Western Cape Province, SA, overshadowed only by diabetes mellitus (7.7\%) and HIV (6.2\%).
ST-segment elevation myocardial infarction (STEMI) contributes significantly to the burden of IHD, and timeous treatment of patients can reduce morbidity and mortality. Primary percutaneous coronary intervention (PCI) is the preferred strategy for the treatment of acute STEMI. If primary PCI is not available, the alternative strategy is to administer a fibrinolytic agent to break down the occlusive thrombus and potentially reperfuse viable myocardium, allowing time for transfer to a PCI centre. The ISIS-2 study ${ }^{[3]}$ found that timeous fibrinolysis led to preservation of left ventricle function by reducing cardiac remodelling. The Division of Cardiology at Tygerberg Hospital, Cape Town, SA follows the guidelines set out by the European Society of Cardiology (ESC), which recommends administration of a fibrinolytic agent within 10 minutes of a STEMI diagnosis if PCI is not available within 60 - 90 minutes (the pharmaco-invasive strategy), followed by referral to a PCI centre. ${ }^{[4]}$

The total time of ischaemia can be divided into the following intervals (Fig. 1):

1. between symptom onset and presentation at a healthcare centre (transport delay)

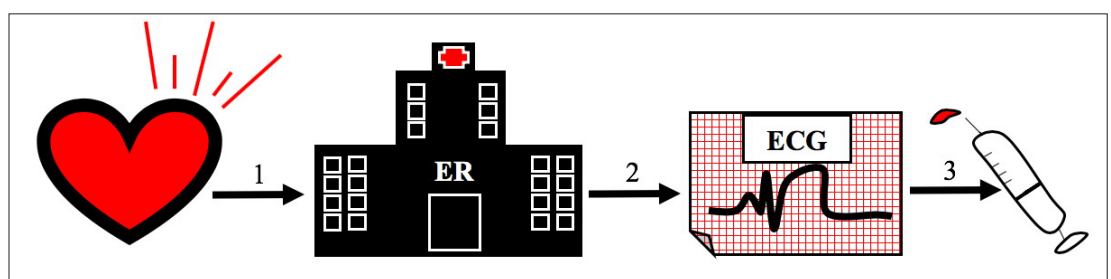

Fig. 1. Time intervals between symptom onset and fibrinolysis. $(E R=$ emergency room; $E C G=$ electrocardiogram.) 
2. between presentation and diagnosis of STEMI (diagnostic delay) 3. between STEMI diagnosis and treatment (treatment delay).

The time between symptom onset and diagnosis can be influenced by a multitude of factors, including recognition of symptoms, transport, as well as access to a healthcare facility. The time between diagnosis and treatment, however, is largely influenced by the competency and efficacy of healthcare staff and can be targeted for improvement if it does not meet guideline-recommended standards. Maximal effect of fibrinolysis is seen when it is administered within 60 minutes of symptom onset. ${ }^{\left[{ }^{[j]}\right.}$ According to a study by Maharaj et al. ${ }^{[6]}$ fibrinolysis is most commonly delayed by the attending doctor seeking advice from senior staff members, difficulties in interpreting the electrocardiogram (ECG) and patients presenting with atypical symptoms.

\section{SA healthcare setting}

Healthcare in SA is comprised of a publicly and privately funded system. The former consists of facilities and staff provided by the National Department of Health, while patients can also opt to be treated in privately funded facilities. A high percentage (84\%) of SA citizens make use of publicly funded healthcare, ${ }^{[7]}$ highlighting the burden placed on these settings.

Tygerberg Hospital is a tertiary academic centre that provides a PCI service to a drainage area spanning large parts of the Western Cape. The geographical distances, coupled with an oftenoverburdened ambulance service, lead to transfer times of $>90 \mathrm{~min}$ utes in most cases. Therefore, the vast majority of STEMI patients are treated initially with a fibrinolytic agent. This emphasises the need for efficient prereferral STEMI treatment. While the ESC STEMI guidelines state a treatment target of 10 minutes, it is anecdotally evident that this target is seldom achieved. We hypothesised that most patients presenting with STEMI are not administered a fibrinolytic agent within 10 minutes, and therefore structured this study to see if this is the case.

\section{Objectives}

The primary aim of this study was to evaluate the time (minutes) between the diagnosis of STEMI and the administration of a fibrinolytic agent. By recording either the time of the diagnostic ECG, or the time of diagnosis as stated by the involved medical doctor, as well as the time of fibrinolysis, we could calculate a mean among our study population and compare that with the proposed standard of care. Making use of statistical analysis, we represent these data as a mean duration (minutes), with a $95 \%$ confidence interval (CI) and a standard deviation (SD) from the mean.

A secondary objective was to assess whether appropriate secondary prevention medication was prescribed on discharge.

\section{Methods \\ Participants and setting}

Tygerberg Hospital is one of two tertiary hospitals in the Western Cape and serves as the referral centre for STEMI patients presenting to peripheral hospitals within a demarcated drainage area. On transfer, these patients are admitted to a dedicated cardiology ward, consisting of an 8-bed coronary care unit and 17 high-care beds with continuous ECG monitoring. We retrospectively identified all patients who were diagnosed with STEMI and admitted to the cardiology ward from 1 March 2017 to 28 February 2018, and included them in our database.

\section{Design}

A retrospective review of case notes was used as research method and the following patient characteristics were evaluated:
- age and gender

- primary presenting centre

- cardiovascular risk factors as per discharge summary (hypertension, diabetes mellitus, previous or current cigarette smoking, dyslipidaemia, family history of premature coronary disease)

- diagnostic accuracy (whether the diagnosis of STEMI was accurate)

- date and time of symptom onset, time of STEMI diagnosis and time of administration of a fibrinolytic agent

- success of fibrinolysis in achieving reperfusion (resolution of chest pain and reduction in ST-segment elevation by $\geq 50 \%$ )

- survival to hospital discharge

- discharge medication.

\section{Data collection}

Tygerberg Hospital's Enterprise Content Management (ECM) system was used to access electronic patient records, including relevant ECGs. The National Health Laboratory Service (NHLS) electronic platform was used for obtaining laboratory variables.

\section{Inclusion and exclusion criteria}

All patients admitted to Tygerberg Hospital between 1 March 2017 and 28 February 2018 with an admission or referral diagnosis of STEMI were included in the study. Patients with no or incomplete clinical case notes on ECM were excluded, as were those erroneously entered into the cardiology admissions book as cases of STEMI.

\section{Outcome measures}

We calculated the time from diagnosis to fibrinolysis in minutes, thereby enabling us to directly compare it with the ESC guideline of 10 minutes.

Secondary prevention forms part of holistic STEMI care. As a secondary outcome, we evaluated whether discharge medication was appropriate. A discharge script consisting of the following 4 drugs was considered to be appropriate:

- angiotensin-converting enzyme (ACE) inhibitor/angiotensin II-receptor blocker (ARB)

- beta-blocker

- aspirin

- statin.

Where a clear reason was stated for the omission of any of the abovementioned medication, secondary prevention was also noted to be appropriate.

\section{Data management and statistical analysis}

Data were imported, cleaned and analysed in Microsoft Office, version 16.0 (Microsoft, USA) using simple descripive statistics. Continious data were presented as either mean and SD or median and interquartile range (IQR), depending on data normality, 95\% CIs were used when appropriate and categorical data were presented as frequency and percentages. Normality of data was determined graphically. Hypothesis testing with non-parametric tests, such as the Mann-Whitney $U$-test, was used where appropriate. Statistical significance was set at $p \leq 0.05$.

\section{Ethical approval}

This study was approved by the Health Research Ethics Committee, Stellenbosch University (ref. no. HREC N18/10/123). Patient anonymity was ensured at all times. There were no ethical concerns of note. 


\section{Results}

Between 1 March 2017 and 28 February 2018, 492 patients with an admission or referral diagnosis of STEMI were identified, of whom 447 were included in the study (Fig. 2).

The mean age of the population was 56 (SD 12) years, and the majority of patients were male $(66.1 \% ; n=299)$. The diagnosis of STEMI was correct in $80.5 \%(n=360)$ of the population; the other patients were either misdiagnosed (4.7\%) - most commonly patients with a non-STEMI - or the STEMI was missed (14.8\%).

A total of 240 (53.7\%) patients presented to a district/provincially funded hospital, while only $17(3.8 \%)$ initially presented to a tertiary facility. A total of 354 (79.2\%) patients were either active or ex-cigarette smokers. The majority of the population (64.9\%) had underlying systemic hypertension, while only $13.9 \%$ of patients admitted to a first-degree relative dying of premature cardiovascular disease (Table 1).

The median time duration between diagnosis and fibrinolysis was 67 (IQR 32.5 122.5) minutes, and 18 patients (6.6\%; 95\% CI 4.0 - 10.3) received fibrinolysis within the recommended 10-minute target (Fig. 3). The median time between symptom onset and fibrinolysis was 270 (IQR 165 490) minutes.

There was no significant difference in time to fibrinolysis between patients treated at public (66 (IQR 30 - 130) minutes) v. private (69 (IQR 28 - 91) minutes) facilities ( $p=0.41)$ (Table 2).

A total of 129 (28.9\%) patients did not receive fibrinolysis; the reasons are as follows (Table 3):

- contraindication to fibrinolysis (7.8\%)

- missed STEMI (54.3\%)

- spontaneous reperfusion of STEMI (10.1\%)

- at Tygerberg Hospital within primary PCI window (10.1\%).

Appropriateness of discharge medication was assessed in 360 patients accurately diagnosed with STEMI; 301 (83.6\%) patients were prescribed appropriate secondary prevention.

Twenty-three (6.4\%) patients correctly diagnosed with STEMI died during their hospital admission. Of the study population, $27(6.0 \%)$ patients died during their hospital stay.

\section{Discussion}

Principal findings

The primary aim of the study was to evaluate whether we are managing patients with
STEMI adequately. As the median time between diagnosis and fibrinolysis was 67 (IQR 32.5 - 122.5) minutes, and only $18(5.7 \%)$ patients were treated within the 10 -minute target, it is safe to state that we are missing the target with some distance. A high patient burden and limitations in the access to fibrinolytic agents are anecdotally some of the challenges of adhering to treatment guidelines, but the data presented in this article prompt more effort to investigate reasons for this significant delay in treatment.
The median time interval between symptom onset and fibrinolysis was 270 (IQR 165 490) minutes. This indicates that the total time of ischaemia was at least 4 hours in more than half of the patients included in the study. The adage of time is muscle holds true and suggests that the majority of the population studied are subject to significant myocardial damage due to the prolonged duration of ischaemia.

The majority of patients presented to public healthcare facilities, but from the abovementioned results we can evaluate

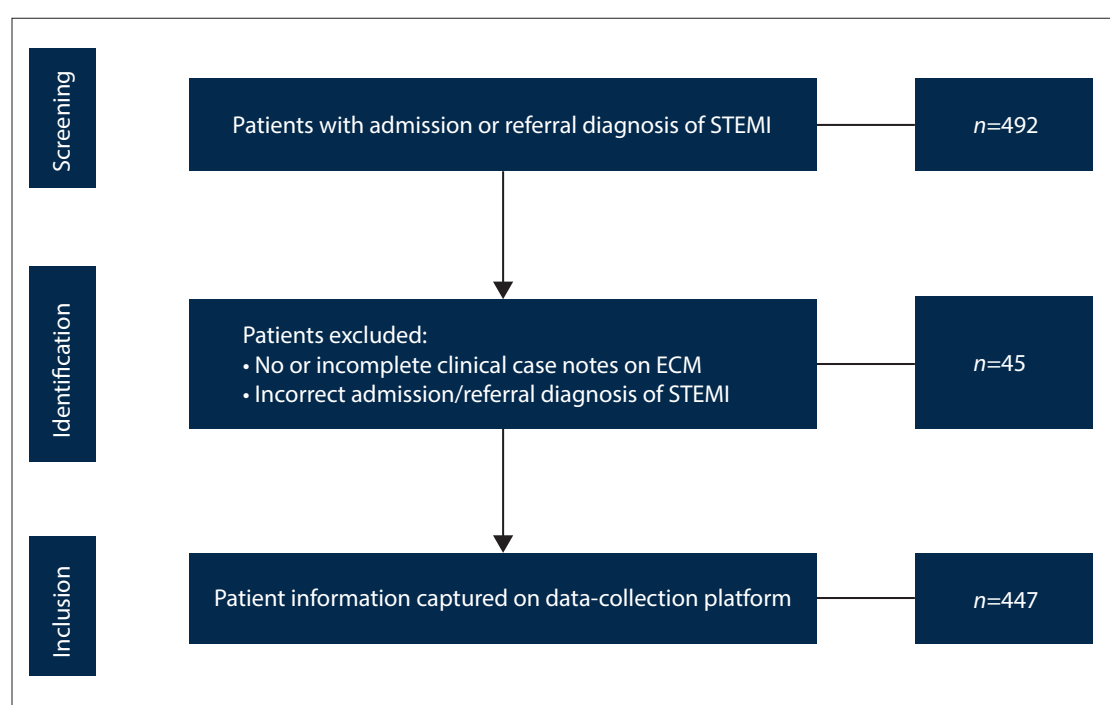

Fig. 2. Patient recruitment and selection. (STEMI = ST-segment elevation myocardial infarction; $E C M=$ Enterprise Content Management.)

\begin{tabular}{ll} 
Table 1. Demographic information & \\
\hline & Results, $\boldsymbol{n}(\%)^{*}$ \\
\hline Age (years), mean (SD) & $56(12)$ \\
Sex & \\
Male & $299(66.1)$ \\
Female & $148(33.9)$ \\
Primary presenting centre & \\
Unknown & $52(11.6)$ \\
Clinic & $51(11.4)$ \\
District/provincially aided hospital & $240(53.7)$ \\
Regional hospital & $53(11.9)$ \\
Tertiary hospital & $17(3.8)$ \\
Private medical facility & $34(7.6)$ \\
Cardiovascular risk factors & \\
Hypertension & $290(64.9)$ \\
Diabetes mellitus & $122(27.3)$ \\
Dyslipidaemia & $158(35.3)$ \\
Smoking history & $354(79.2)$ \\
Family history & $62(13.9)$ \\
Diagnosis & \\
Correct diagnosis of STEMI & $360(80.5)$ \\
Incorrect diagnosis/diagnoses other than STEMI & $21(4.7)$ \\
Correct diagnosis of STEMI, but missed & $66(14.8)$ \\
SD = standard deviation; STEMI = ST-segment elevation myocardial infarction. & \\
${ }^{*}$ Unless otherwise specified. & \\
&
\end{tabular}




\begin{tabular}{|c|c|}
\hline & Median (IQR), $\min ^{\dagger}$ \\
\hline Patients who received fibrinolysis, $n$ (\%) & $318(71.1)$ \\
\hline Documented time to fibrinolysis & $271(85.2)$ \\
\hline$\leq 10$ & $18(6.6)$ \\
\hline $11-30$ & $46(17.0)$ \\
\hline $31-60$ & $67(24.7)$ \\
\hline $61-240$ & $115(42.4)$ \\
\hline$>240$ & $25(9.2)$ \\
\hline Undocumented time to fibrinolysis & $47(14.8)$ \\
\hline Successful fibrinolysis, $n$ (\%) & $234(73.6)$ \\
\hline \multicolumn{2}{|l|}{ Time interval } \\
\hline $\begin{array}{l}\text { Between diagnosis and fibrinolysis in all } \\
\text { centres }\end{array}$ & $67(32.5-122.5)$ \\
\hline Between symptom onset and fibrinolysis & $270(165-490)$ \\
\hline $\begin{array}{l}\text { Between diagnosis and fibrinolysis in } \\
\text { private centres }\end{array}$ & $69(28-91)$ \\
\hline $\begin{array}{l}\text { Between diagnosis and fibrinolysis in } \\
\text { public centres }\end{array}$ & $66(33-130)$ \\
\hline $\begin{array}{l}\text { Difference between patients in private } \\
\text { and public centres }\end{array}$ & $3^{*}$ \\
\hline $\begin{array}{l}\text { IQR }=\text { interquartile range. } \\
{ }^{*} p=0.41 . \\
\text { 'Unless otherwise specified. }\end{array}$ & \\
\hline
\end{tabular}

Table 3. Discharge information

\begin{tabular}{ll}
\hline & Results, $\boldsymbol{n}$ (\%) \\
\hline Discharge medication & \\
Appropriate discharge medication & $301(83.6)$ \\
Inappropriate discharge medication & $59(10.3)$ \\
Deceased & $23(6.3)$ \\
Aspirin & \\
$\quad$ Prescribed appropriately & $382(95.7)$ \\
Omitted without reason & $15(3.8)$ \\
Contraindicated & $2(0.5)$ \\
Statin & \\
Prescribed appropriately & $376(94.2)$ \\
Omitted without reason & $23(5.8)$ \\
Contraindicated & $0(0)$ \\
ACE inhibitor/ARB & \\
Prescribed appropriately & $384(96.2)$ \\
Omitted without reason & $13(3.3)$ \\
Contraindicated & $2(0.5)$ \\
Beta-blocker & \\
Prescribed appropriately & $379(95.0)$ \\
Omitted without reason & $17(4.3)$ \\
Contraindicated & $3(0.8)$ \\
Mortality & \\
Overall study population & $27(6.0)$ \\
Patients correctly diagnosed with STEMI & $23(6.4)$ \\
& \\
ACE = angiotensin-converting enzyme; ARB = angiotensin II-receptor blocker; \\
STEMI = ST-segment elevation myocardial infarction. \\
$\quad$
\end{tabular}

whether patients referred to Tygerberg Hospital after receiving fibrinolysis at private healthcare facilities received treatment more timeously. In private healthcare facilities, the median time between diagnosis and fibrinolysis was 69 (IQR 18 - 91) minutes compared with 66 (IQR 33 - 130) minutes in public healthcare facilities. This difference of 3 minutes $(p=0.41)$ is not statistically significant and

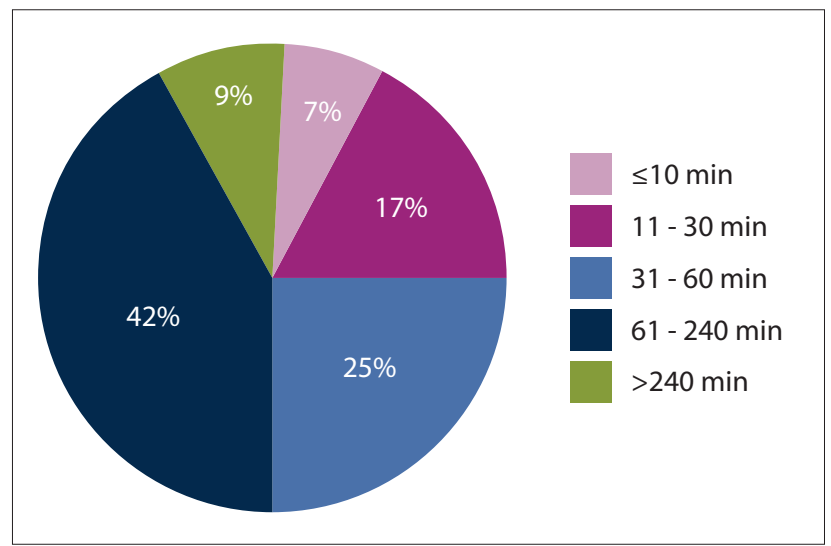

Fig. 3. Patients receiving fibrinolysis - time intervals.

cannot be interpreted as being accurate of the larger population, as the data of only 21 patients were analysed. Nonetheless, it does give some indication that the therapeutic delay of STEMI is not only limited to public healthcare centres.

Comprehensive management of STEMI not only includes primary PCI or fibrinolysis, but also the administration of adequate secondary preventive drugs. In a study by Griffiths et al. ${ }^{\left[{ }^{[8]}\right.}$ the importance of secondary preventive measures after acute coronary syndrome is outlined, i.e. smoking cessation and administration of a combination of 4 drugs: aspirin, beta-blocker, ACE inhibitor or ARB, and a statin. In this study, it was found that $71 \%$ of patients were discharged on the appropriate medication regimen, while only $48 \%$ were taking the medication at their 6-month follow-up visit. This serves as a reminder that not only does emergency treatment of STEMI reduce the disease burden caused by IHD, but that emphasis is required on the importance of secondary prevention and longer-term management. At discharge from Tygerberg Hospital, 301 (83.6\%) patients correctly diagnosed with STEMI received adequate discharge medication.

With regard to the abovementioned primary and secondary outcomes, one can conclude that more emphasis should be placed on timeous treatment of STEMI in healthcare centres prior to referral to cardiac units, given the inevitable delays in transportation of patients. Medical personnel should familiarise themselves with early recognition of symptoms, accurate diagnosis and appropriate management of acute STEMI to improve management of IHD. By reducing the delay in treatment, we can reduce the burden of IHD on the individual and on the greater healthcare system in SA.

\section{Study strengths and limitations}

The data presented in this article are comprehensive with regard to the population size, geographical involvement of the drainage area of a tertiary referral centre and inclusion of patients diagnosed over 12 months. This information enabled us to accurately analyse STEMI treatment in the involved facilities and allows extrapolation of results to the Western Cape and the greater SA.

Further data from the private healthcare sector are needed to comment with more certainty on the correlation between treatment times in public and private healthcare facilities. The study findings must be seen in view of the limited data, but still offer some interesting results.

Lastly, there is a discrepancy in the guidelines set by the ESC and the American Heart Association/American College of Cardiology (AHA/ACC) in that the latter recommends fibrinolysis within 30 minutes of diagnosis of STEMI,${ }^{[9]}$ as opposed to the ESC's guideline of 10 minutes. The AHA/ACC guideline moves the target significantly closer to the results obtained in this study. 


\section{Unanswered questions and recommendations for future studies}

The results of this study are useful, as they enable evaluating whether the involved healthcare facility adheres to treatment guidelines. There are multiple factors that influence the therapeutic delay between diagnosis and treatment, and without this knowledge one cannot address the problems identified. To improve the treatment of STEMI, it will therefore be interesting and invaluable to know what modifiable factors are involved in prolonging the duration between diagnosis and fibrinolysis.

\section{Conclusions}

Knowing that IHD is a major contributor to mortality in the Western Cape and the greater SA, it is becoming more important to treat patients presenting with acute STEMI effectively. In centres within the drainage area of Tygerberg Hospital, we found that the median duration between diagnosis of STEMI and fibrinolysis was 67 (IQR 32.5 - 122.5) minutes. This is in contrast to the treatment guideline of 10 minutes of the ESC. It is therefore imperative that factors affecting treatment delay be investigated and addressed to improve patient outcomes and decrease the burden of IHD on the healthcare system of SA.

\section{Declaration. None}

Acknowledgements. We would like to thank Jana Labuschagne, Franco Denkema, Jessica Human and Nicola Duvenage for their contribution to the data collection, as well as Anke Beyers for her assistance with editing of the text. To the Division of Epidemiology and Biostatistics, Department of Global Health, Stellenbosch University, we owe much appreciation for their input and guidance with statistical analysis of the data. We also extend our gratitude to the Division of Cardiology, Department of Medicine, Faculty of Medicine and Health Sciences, Stellenbosch
University and Tygerberg Hospital, for their generosity in making funds available to enable the publication of this research article.

Author contributions. BDB: wrote the protocol, captured data and wrote the article; TJ: captured data, edited and reviewed the article; BG: planned, edited and reviewed the article; AFD: edited and reviewed the article.

Funding. Funds involved in the data collection, ethics committee application and publication of the study were granted by the Division of Cardiology Research Fund, Stellenbosch University and Tygerberg Hospital.

Conflicts of interest. None.

1. World Health Organization. Global Health Estimates 2015: Deaths by Cause, Age, Sex, by Country and by Region. Geneva: WHO, 2018. https://www.who.int/healthinfo/global_burden_disease/estimates/ en/indexl.html (accessed 22 January 2019).

2. Statistics South Africa. Mortality and Causes of Death in South Africa, 2016: Findings From Death Notification. Pretoria: StatsSA, 2019. http://www.statssa.gov.za/publications/P03093/P030932016.pdf (accessed 21 February 2019).

3. Baigent C. Randomised trial of intravenous streptokinase, oral aspirin, both, or neither amoung 17187 cases of suspected acute myocrdial infarction: ISIS-2. Lancet 1988;332(8607):349-360. https://doi. org $/ 10.1136 / \mathrm{bmij} .316 .7141 .1337$

4. Ibanez B, James S, Agewall S, et al. 2017 ESC guidelines for the management of acute myocardial infarction in patients presenting with ST-segment elevation. Eur Heart J 2017;39(2):119-177. https:/ infarction in patients presenting

5. Boersma E, Maas A, Deckers J, Simoons M. Early thrombolytic treatment in acute myocardial Boersma E, Maas A, Deckers J, Simoons M. Early thrombolytic treatment in acute myocardial
infarction: Reappraisal of the golden hour. Lancet 1996;348(9030):771775. https://doi.org/10.1016/ infarction: Reappraisal of

6. Maharaj R, Geduld H, Wallis L. Door-to-needle time for administration of fibrinolytics in acute myocardial infarction in Cape Town. S Afr Med J 2012;102(4)249-252. https://doi.org/10.7196/ SAMJ.5188

7. African Institute for Health and Leadership Development. Minimum data sets for human resources for health and the surgical workforce in South Africa's health system: A rapid analysis of stock and migration. 2015. https://www.who.int/workforcealliance/031616south_africa_case_studiesweb.pd (accessed 21 February 2020).

8. Griffiths B, Lesosky M, Ntsekhe M. Self-reported use of evidence-based medicine and smoking cessation 6 - 9 months after acute coronary syndrome: A single-centre perspective. S Afr Med 2014;104(7):483-487. https:///doi.org/10.7196/SAMJ.7798

9. O'Connor RE, Brady W, Brooks SC, et al. Acute coronary syndromes: 2010 American Heart Aconnor RE, Brady W, Brooks $\mathrm{SC}$, et al. Acute coronary syndromes: 2010 American Heart
Association guidelines for cardiopulmonary resuscitation and emergency cardiovascular care. Circulation 2010;122(suppl 3):S787-S817. https://doi.org/10.1161/CIRCULATIONAHA.110.971028

Accepted 27 September 2019. 